\title{
Trajectories of health-related quality of life among people with a physical disability and/or chronic disease during and after rehabilitation: a longitudinal cohort study
}

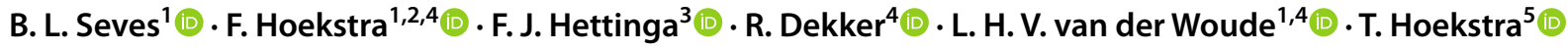

Accepted: 19 September 2020 / Published online: 28 September 2020

(c) The Author(s) 2020

\begin{abstract}
Purpose To identify Health-related Quality of Life (HR-QoL) trajectories in a large heterogeneous cohort of people with a physical disability and/or chronic disease during and after rehabilitation and to determine which factors before discharge are associated with longitudinal trajectory membership.

Methods A total of 1100 people with a physical disability and/or chronic disease were included from the longitudinal cohort study Rehabilitation, Sports and Active lifestyle. All participants participated in a physical activity promotion programme in Dutch rehabilitation care. HR-QoL was assessed using the RAND-12 Health Status Inventory questionnaire at baseline (T0: 3-6 weeks before discharge) and at 14 (T1), 33 (T2) and 52 (T3) weeks after discharge from rehabilitation. A data-driven approach using Latent Class Growth Mixture modelling was used to determine HR-QoL trajectories. Multiple binomial multivariable logistic regression analyses were used to determine person-, disease- and lifestyle-related factors associated with trajectory membership.

Results Three HR-QoL trajectories were identified: moderate $(N=635)$, high $(N=429)$ and recovery $(N=36)$. Trajectory membership was associated with person-related factors (age and body mass index), disease-related factors (perceived fatigue, perceived pain and acceptance of the disease) and one lifestyle-related factor (alcohol consumption) before discharge from rehabilitation.

Conclusions Most of the people who participated in a physical activity promotion programme obtained a relatively stable but moderate HR-QoL. The identified HR-QoL trajectories among our heterogeneous cohort are disease-overarching. Our findings suggest that people in rehabilitation may benefit from person-centred advice on management of fatigue and pain (e.g. activity pacing) and the acceptance of the disability.
\end{abstract}

Keywords Quality of life · Active lifestyle $\cdot$ Health promotion - Rehabilitation · Latent class growth (mixture) models Activity pacing

Electronic supplementary material The online version of this article (https://doi.org/10.1007/s11136-020-02647-7) contains supplementary material, which is available to authorized users.

B. L. Seves

b.l.seves@umcg.nl

1 Center for Human Movement Sciences, University Medical Center Groningen, University of Groningen, Groningen, The Netherlands

2 School of Health and Exercise Sciences, University of British Columbia Okanagan, Kelowna, BC, Canada

3 Department of Sport, Exercise and Rehabilitation, Northumbria University, Newcastle, UK
4 Department of Rehabilitation Medicine, University Medical Center Groningen, University of Groningen, Groningen, The Netherlands

5 Department of Health Sciences and Amsterdam Public Health Research Institute, Vrije Universiteit Amsterdam, Amsterdam, The Netherlands 


\section{Introduction}

Improving health-related quality of life (HR-QoL) is one of the key objectives in today's rehabilitation practice. When evaluating rehabilitation treatments, interventions taking place in rehabilitation practice and policy in health care, HR-QoL is often used as an outcome measure [1,2]. In people with a physical disability and/or chronic disease, HR-QoL during rehabilitation is lower than in the nondisabled population [3]. More importantly, after rehabilitation, low levels of HR-QoL are commonly reported in people with a physical disability and/or chronic disease [4-6], and HR-QoL is poorer compared to a healthy reference population [7]. Low levels of HR-QoL are associated with secondary health conditions (e.g. fatigue, pain, obesity and cardiovascular diseases), whereby preventing secondary health conditions among this target population is an important step towards sustainable health [8] and healthy ageing. Furthermore, low levels of HR-QoL are associated with inactivity and sedentary behaviour in healthy adults [9, 10]. Also, previous literature found that physical activity is positively associated with all components of HR-QoL, except for mental health in people after rehabilitation [7]. Physical activity promotion programmes in rehabilitation care could have positive impact on improving HR-QoL by reducing secondary health conditions during but also after treatment has finished $[4,11,12]$.

According to the literature, there is large heterogeneity in HR-QoL development among people with disabilities [7]. Therefore, investigating HR-QoL by looking at average levels within the sample is not as useful as by investigating subgroups with distinct developmental trajectories of HR-QoL. Previous studies already identified several trajectories of HR-QoL in people during or after rehabilitation from breast cancer or stroke, which were related to the proposed characteristic trajectories of level of dysfunction: high, recovery, decline and low HR-QoL [13-15].

Cross-sectional research into the determinants of HRQoL has found that personal factors (e.g. age and gender) are associated with HR-QoL in people with heart diseases [16] and in aneurysmal subarachnoid haemorrhage (SAH) survivors [17]. Psychosocial factors (e.g. self-efficacy, acceptance, passive coping) are associated with longitudinal HR-QoL in breast cancer survivors [13], in people post stroke [18] and in SAH survivors [17]. Psychological factors (e.g. depression, anxiety and fatigue) predict longitudinal trajectory membership of HR-QoL trajectories in people post stroke [14] and in SAH survivors [19] and predict cross-sectional HR-QoL in people with renal cell carcinoma [20]. Disease-related factors such as disease awareness in people after traumatic brain injury [21] and having comorbidities in people with renal cell carcinoma
[20] were associated with, respectively, cross-sectional and longitudinal HR-QoL.

Most rehabilitation treatments or interventions to promote physical activity have not been evaluated for effectiveness on sustainable HR-QoL after rehabilitation treatment [2, 22]. So far, very little attention has been paid to a disease-overarching mechanism in the heterogeneous course of HR-QoL after rehabilitation. Previous research on HR-QoL development usually focussed on specific disease populations. The current longitudinal study provides an important opportunity to advance the understanding of the course of HR-QoL after rehabilitation, by undertaking a disease-overarching prospective analysis of HR-QoL. In addition, more insight into relevant determinants, such as person-, disease- and lifestyle-related factors is needed to identify vulnerable people with a physical disability and/or chronic disease at risk to experience a reduced HR-QoL after discharge already in the early stages of rehabilitation. These determinants can be non-modifiable (e.g. gender, age, severity of the disability) or modifiable (e.g. physical activity behaviour, acceptance of the disability, the use of tobacco and alcohol). Modifiable factors should be targeted by rehabilitation professionals, to improve patients' HR-QoL. The findings of this study may support the need for more person-centred care to help people to obtain and maintain sustainable high levels of HR-QoL after rehabilitation.

Therefore, the purposes of this study were (1) to identify trajectories of HR-QoL up to 1 year after discharge from rehabilitation in people with a physical disability and/or chronic disease and (2) to determine person-, disease- and lifestyle-related factors before discharge from rehabilitation that are associated with longitudinal trajectory membership.

\section{Methods}

\section{Context}

The current study is part of the multicentre longitudinal cohort study Rehabilitation, Sports and Active lifestyle (ReSpAct) that was initiated to evaluate the nationwide programme Rehabilitation, Sports and Exercise (RSE; Dutch: 'Revalidatie, Sport en Bewegen') [23, 24]. The RSE programme has been implemented in eighteen rehabilitation institutions in the Netherlands (twelve rehabilitation centres and six rehabilitation departments of hospitals). The RSE programme aims to stimulate an active lifestyle during the rehabilitation period and to guide people with a physical disability and/or chronic disease in maintaining a physically active lifestyle in the home setting after discharge from rehabilitation [23, 24]. Participants of the RSE programme were referred to a sports counselling counter 3 to 6 weeks before discharge from rehabilitation for a face-to-face consultation 
with a sports counsellor, followed by four telephone-based counselling sessions up to 13 weeks after discharge from rehabilitation [23, 24]. All sessions were based on motivational interviewing [25] (see Online Resource 2 for a schematic overview of the RSE programme and the ReSpAct study).

Participants were included in the ReSpAct study from May 2013 to August 2015. Participants were monitored with questionnaires at given regular measurement times: at baseline (T0: 3-6 weeks before discharge) and 14 (T1), 33 (T2) and 52 (T3) weeks after discharge from rehabilitation (Online Resource 2). The study was approved by the ethics committee of the Center for Human Movement Sciences of the University Medical Center Groningen (reference: ECB/2013.02.28_1). All participants voluntarily participated after signing an informed consent.

\section{Study population}

Inclusion criteria were: (1) being at least 18 years of age, (2) having a chronic disease or physical disability (e.g. stroke, heart failure, Parkinson's disease, spinal cord injury), (3) receiving inpatient or outpatient rehabilitation care or treatment at one of the participating rehabilitation departments or institutions, (4) participating in the RSE programme [24] and (5) filling in the RAND-12 Health Status Inventory (RAND-12) at two or more measurement occasions. Participants were excluded if they were not able to complete the questionnaires, even with help, or were participating in another physical activity stimulation programme.

\section{HR-QoL}

HR-QoL was assessed by using the self-reported RAND12 questionnaire [26], an adapted, abbreviated version of the RAND-36 Health Status Inventory (RAND-36) [27]. The RAND-12 contains at least one item from each of the eight subscales of the RAND-36, so that it adequately represents the wide range of relevant aspects of health status [28]. Six items of the RAND-12 contribute to the physical health composite (how health limits a person in activities, or how a person's physical health causes problems with work or other activities) and six other items contribute to the mental health composite (how a person feels and how a person's mental health causes problems with work or other activities) $[27,28]$. All twelve items contribute to the general health composite, which represents all relevant aspects of health status [28]. We used an age-corrected general health composite score for this study [27]. A higher score on the RAND-12 indicated better HR-QoL. Because the RAND-12 only contains twelve items of the RAND-36 (range 0-100), scores on the RAND-12 range from 0 to 65 . We found good reliability (internal consistency) of the RAND-12 based on the study sample at T0 (Cronbach's $\alpha=0.85, N=974)$, at T1 (Cronbach's $\alpha=0.87, N=957$ ), at T2 (Cronbach's $\alpha=0.88$, $N=861$ ) and at T3 (Cronbach's $\alpha=0.88, N=780$ ). Previous literature supports acceptable construct validity and test-retest reliability of the RAND-12 in among others clinical populations $[28,29]$.

\section{Person-, disease- and lifestyle-related factors}

All independent variables were measured at baseline (T0: 3-6 weeks before discharge). Person-related factors included gender, age, body mass index (BMI) and level of education, which was dichotomized into low (up to completed secondary education) and high (completed applied University or higher) to make it internationally comparable.

Disease-related factors included the type of disease divided into eight categories: musculoskeletal disease, amputation, brain disorder (e.g. stroke or other non-congenital brain defects), spinal cord injury, other neurologic disease, organ disease, chronic pain and other diseases. Also, disease-related factors included the number of comorbidities dichotomized into no comorbidities and one or more comorbidities, because this variable included all diseases and disabilities reported by a participant. The level of acceptance of the disability or disease was assessed on a four-point Likert scale (1-4, no acceptance to complete acceptance), with a higher score indicating better acceptance of the disability or disease. The level of acceptance was dichotomized into no (no or little acceptance) and yes (acceptance to a large extent or completely), because when entering the level of acceptance as categorical variable in the logistic regression, we found that the odds ratios (ORs) did not linearly increased/decreased. Perceived fatigue was assessed with the 9-item Fatigue Severity Scale (FSS) [30], which is a valid and reliable questionnaire to determine the impact of perceived fatigue in clinical populations (in people with systematic lupus erythematosus $r_{\text {validity }}=0.81$ and $r_{\text {reliability }}=0.89$, and in people with multiple sclerosis $r_{\text {validity }}=0.47$ and $r_{\text {reliability }}=0.81$ ) [30-32]. The FSS score ranges from 1 to 7 , with a higher score indicating more perceived fatigue [30]. We found good reliability (internal consistency) of the FSS based on the study sample at T0 (Cronbach's $\alpha=0.91, N=1044)$. The FSS includes items like "Exercise brings on my fatigue." and "I am easily fatigued" [30]. The level of perceived pain was assessed on a sixpoint Likert scale (1-6, from no pain to severe pain), with a higher score indicating more perceived pain. The level of pain was dichotomized into no (no to light pain: score 1-3) and yes (moderate to severe pain: score: 4-6), because when entering perceived pain as categorical variable in the logistic regression, we found that the ORs did not linearly increased/decreased. Also, too few people reported severe pain (perceived pain $=6$ ). 
Lifestyle-related factors included the dichotomous variables smoking and alcohol use ("Do you smoke currently?" and "Do you consume alcohol currently?": yes or no). In addition, the total minutes of physical activity per week was assessed by using the Adapted Short Questionnaire to Assess Health-enhancing physical activity (Adapted-SQUASH), a 19 -item self-reported recall questionnaire. In a previous study, the Adapted-SQUASH has been shown to be a sufficiently reliable (intraclass correlation coefficient $=0.76$, $p<0.001)$ and valid-compared to the Actiheart activity monitor-(intraclass correlation coefficient $=0.22$, $p=0.027$ ) questionnaire to determine self-reported physical activity in a similar sample (people with a physical disability and/or chronic disease) [33]. The Adapted-SQUASH is pre-structured in four main domains outlining types and settings of activity: 'commuting traffic', 'activities at work and school', 'household activities' and 'leisure time activities' including 'sports activities' [34]. The SQUASH [34] was adapted to make the questionnaire more applicable for this population (Adapted-SQUASH), as described in the study protocol of the ReSpAct study [24]. First, the items 'wheeling in a wheelchair' and 'handcycling' were added in the domains 'commuting activities and leisure time' and 'sports activities'. Second, the self-reported intensity of the activity was categorised in 'light', 'moderate' and 'vigorous', instead of 'slow', 'moderate' and 'fast'. Third, a large range of adapted sports (e.g. wheelchair basketball/rugby/ tennis) were included for the item 'sports activities'. Lastly, in the examples of different sports 'tennis' was replaced by '(wheelchair) tennis'. Information on sports participation (yes/no) was obtained from the Adapted-SQUASH. If the participant reported to perform at least one sports activity per week, than they were coded as 'yes', if not as 'no'.

\section{Statistical analysis}

Analyses were conducted in a two-step approach. First, trajectories of HR-QoL during and after rehabilitation among participants with two or more valid measurements over time were identified using Latent Class Growth Mixture (LCGM) modelling with quadratic (assuming non-linear change over time), linear (assuming linear change over time) and latent class analyses (lca) models [35], using the Mplus software program 7.11. The choice for linear and quadratic models was made based on previous research [14], showing trajectories of HRQoL to be both linear as well as quadratic (non-linear). Additionally, latent class analyses were conducted for descriptive purposes. These analyses gave us insight in the (heterogeneity of) patterns of change in HR-QoL without a priori assuming a trajectory shape. LCGM models are regression-based models that assume that individuals in the sample do not necessarily come from one underlying population but might come from multiple underlying (or latent) subpopulations. LCGM modelling aims to find the optimal number and characteristics of these subpopulations. Common, stepwise modelling strategies were applied [35], using the Guidelines for Reporting on Latent Trajectory Studies (GRoLTS) as well [36]. A one-class model was first determined, thus assuming one underlying population, and subsequently more classes were added one at a time and model fit indices were inspected. The optimal number of classes was determined according to the following model fit criteria: (1) a lower Bayesian Information Criterion (BIC), where a difference of 10 points lower is usually regarded as sufficient improvement [37], (2) a higher entropy (range from 0 to 1 ), a standardised measure of how accurately individuals' trajectories are classified, where higher values indicate better classification [38, 39] and (3) average posterior probabilities of $\geq 0.80$ [35]. The choice for the optimal number of classes was additionally made considering clinical interpretation (rejecting solutions that do not make clinical sense) and class size. Finally, individuals were classified into their most likely class based on their posterior probability.

Second, multiple binomial multivariable logistic regression analyses were performed to assess associations between the previously described person-, disease- and lifestyle-related factors and trajectory membership using version 24 of the Statistical Package for the Social Science (SPSS). The outcome of the LCGM modelling, the nominal variable of trajectory membership, was used as dependent variable.

Independent variables at baseline were all entered block wise (block 1: person-related factors, block 2: disease-related factors and block 3: lifestyle-related factors) in multivariable models. Descriptive statistics of these variables were analysed at baseline. Assumptions of normality and linearity were checked. The continuous independent variables age, BMI, fatigue, and physical activity/week were standardised. Results of the multiple binomial multivariable logistic regression analyses are presented as odds ratio (OR) and corresponding 95\% confidence interval (CI). Because three comparisons between two trajectories were needed to compare all HR-QoL trajectories, a Bonferroni-corrected $p$-value, to correct for multiple testing, of $0.017(0.05 / 3=0.017)$ was used to give a $95 \%$ probability of correctly concluding not to reject the null hypothesis [40].

To facilitate transparency and reproducibility, additional information is available on: (a) the dataset of the HR-QoL (Online Resource 1) and (b) the Mplus syntax of the LCGM modelling and the SPSS syntax of the multiple binomial multivariable logistic regression analyses (Online Resource 2).

\section{Results}

\section{Characteristics of participants}

In total 1100 participants were included in this study. Participants had an average age of $51.0 \pm 13.5$ years and $52.0 \%$ were 
female. The three most common disease groups were brain disorder $(26.0 \%, N=286)$, musculoskeletal disease $(18.1 \%$, $N=199)$ and chronic pain $(15.6 \%, N=172)$ (Table 1$)$.

Based on descriptive characteristics at baseline (Table 1), participants excluded for the LCGM modelling analyses were on average more often female, younger, lower educated, lived less independently, had worse acceptance of their disease, perceived more fatigue, smoked less, received less counselling moments and had lower levels of HR-QoL. Descriptive characteristics at baseline were missing of
Table 1 Participants' descriptive statistics at baseline for participants included $(N=1100)$ and excluded $(N=617)$ in the latent class growth mixture modelling analyses

\begin{tabular}{|c|c|c|}
\hline Characteristic & $\begin{array}{l}\text { Included in LCGMM } \\
\text { Mean } \pm \text { SD or } \%(N)\end{array}$ & $\begin{array}{l}\text { Excluded for LCGMM } \\
\text { Mean } \pm \mathrm{SD} \text { or } \%(N)\end{array}$ \\
\hline \multicolumn{3}{|l|}{ Personal-related factors } \\
\hline Gender ( $\%$ female) & $52.0(572)$ & $57.8(358)^{*}$ \\
\hline Age in years & $51.0 \pm 13.5$ & $47.8 \pm 13.9^{* *}$ \\
\hline Body mass index $\left(\mathrm{kg} / \mathrm{m}^{2}\right)$ & $27.2 \pm 5.5$ & $27.6 \pm 6.2$ \\
\hline 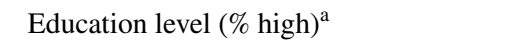 & $24.5(270)$ & $11.5(71)^{*}$ \\
\hline Living situation (\% independent) & $88.7(976)$ & $53.0(328)^{*}$ \\
\hline \multicolumn{3}{|l|}{ Disease-related factors } \\
\hline \multicolumn{3}{|l|}{ Disease group } \\
\hline Brain disorders & $26.0(286)$ & $27.1(168)$ \\
\hline Musculoskeletal disease & $18.1(199)$ & $19.2(119)$ \\
\hline Chronic pain & $15.6(172)$ & $17.8(110)$ \\
\hline Neurologic disease & $15.5(171)$ & $12.1(75)$ \\
\hline Organ disease & $12.0(132)$ & $10.7(66)$ \\
\hline Amputation & $4.5(50)$ & $4.4(27)$ \\
\hline Other symptoms & $4.0(44)$ & $3.1(19)$ \\
\hline Spinal cord injury & $2.8(31)$ & $4.4(27)$ \\
\hline Acceptance (\% yes) & $54.3(597)$ & $28.4(176)^{*}$ \\
\hline Comorbidities ( $\%$ yes) & $41.3(454)$ & $28.1(174)$ \\
\hline Fatigue (FSS score) & $4.3 \pm 1.5$ & $4.5 \pm 1.5^{*}$ \\
\hline Pain (\% yes) & $46.2(508)$ & 25.7 (159) \\
\hline \multicolumn{3}{|l|}{ Lifestyle-related factors } \\
\hline Smoking (\% yes) & $16.4(180)$ & $13.7(85)^{*}$ \\
\hline Alcohol use (\% yes) & $39.1(430)$ & $18.6(115)$ \\
\hline Total minutes of PA/week & $1081.1 \pm 919.5$ & $1120.8 \pm 966.8$ \\
\hline Sports participation (\% yes) & $54.5(600)$ & $45.6(282)$ \\
\hline \multicolumn{3}{|l|}{ Institutional level } \\
\hline Treatment form ( $\%$ outpatient $)^{\mathrm{c}}$ & $90.4(994)$ & $89.0(551)$ \\
\hline Treatment context (\% hospital) & $28.1(309)$ & $26.2(162)$ \\
\hline $\begin{array}{l}\text { Amount of physical activity counselling } \\
\text { moments after rehabilitation }{ }^{\mathrm{d}}\end{array}$ & $2.6 \pm 1.4$ & $2.1 \pm 1.5^{*}$ \\
\hline \multicolumn{3}{|l|}{ Health-related quality of life (RAND-12) } \\
\hline Mental health composite & $40.3 \pm 9.4$ & $38.5 \pm 9.3 *$ \\
\hline Physical health composite & $36.2 \pm 10.3$ & $33.6 \pm 9.4 * *$ \\
\hline General health composite & $37.2 \pm 9.3$ & $34.7 \pm 8.8^{* *}$ \\
\hline
\end{tabular}

$S D$ standard deviation, $N$ number of participants, LCGMM latent class growth mixture modelling, FSS Fatigue Severity Scale, $P A$ Physical activity

${ }^{a}$ Completed applied University or higher

${ }^{b}$ Percentage of participants with one or more comorbidities

${ }^{\mathrm{c}}$ Treatment form includes outpatient and inpatient

${ }^{\mathrm{d}}$ Participants in the Rehabilitation, Sports and Exercise programme received four telephone-based counselling sessions with a sports counsellor

$*$ and $* *$ The characteristic is significantly different $(* p<0.05, * * p<0.01)$ between the participants included and excluded for the LCGMM based on independent sample t-tests for continuous variables and based on Chi-square tests for categorical variables 
around 250 excluded participants, which might give skewed descriptive characteristics.

\section{HR-QoL trajectories}

The results of the fit indices for quadratic, linear and lca models with one to six trajectories of HR-QoL are presented in Table 2. Comparing these models with the model fit criteria alone proved to be complicated, as the model fit criteria were not always in agreement, which is a common finding in LCGM modelling [41]. After careful consideration, we chose the three-class quadratic model as the optimal model in this sample, although the average posterior probabilities were slightly below 0.80 , indicating possibly less distinct trajectories and subsequent fuzzy classification, yet it avoids inclusion of an extremely small class, as is the case in the four-class and five-class quadratic models. The three-trajectory model consisted of two large and stable, but distinctly different trajectories: moderate $(N=635,55.1 \%)$ and high $(N=429 ; 40.9 \%)$ trajectory. In addition, one smaller intermediate trajectory is provided, which increases between 3 and 6 weeks before discharge from rehabilitation and
33 weeks post rehabilitation and then stabilises (i.e. recovery) $(N=36 ; 4.0 \%)$ (Fig. 1$)$.

Descriptive statistics of the mental, physical and general health composites for the three trajectories at each measurement time are presented in Table 3. Overall, mental health followed the same but higher course and physical health followed the same but lower course compared to general health. Supplementary figures are given in Online Resource 2 , including estimated mean trajectories for each model, estimated means with individual trajectories for each latent class and the estimated with observed means for the final model. Although the plots with estimated means with individual trajectories for each latent class show large heterogeneity in individual trajectories of HR-QoL, all individual trajectories follow the same growth pattern over time for each latent class.

\section{Determinants of HR-QoL trajectories}

Descriptive statistics of possible determinants before discharge from rehabilitation for the HR-QoL trajectories are presented in Table 4. Multiple binomial multivariable logistic regression analyses were performed to determine
Table 2 Fit indices for quadratic, linear and lca models with 1-6 trajectories of HR-QoL

\begin{tabular}{|c|c|c|c|c|c|c|c|c|c|}
\hline \multicolumn{10}{|c|}{ Health-related quality of life } \\
\hline \multirow[t]{2}{*}{ Number of classes } & \multirow[t]{2}{*}{$\mathrm{BIC}$} & \multirow[t]{2}{*}{ Entropy } & \multirow{2}{*}{$\begin{array}{l}\text { Average posterior } \\
\text { probability (min- } \\
\max )\end{array}$} & \multicolumn{6}{|c|}{$\begin{array}{l}\text { Number of participants in each trajec- } \\
\text { tory class }\end{array}$} \\
\hline & & & & 1 & 2 & 3 & 4 & 5 & 6 \\
\hline \multicolumn{10}{|l|}{ Quadratic analyses } \\
\hline 1 & $24,301.36$ & NA & 1.0 & 1100 & & & & & \\
\hline 2 & $24,227.49$ & .87 & $.90(.83-.97)$ & 1058 & 42 & & & & \\
\hline 3 & $24,198.33$ & .61 & $.79(.76-.83)$ & 36 & 635 & 429 & & & \\
\hline 4 & $24,201.32$ & .67 & $.83(.77-.95)$ & 2 & 640 & 42 & 416 & & \\
\hline 5 & $24,196.12$ & 69 & $.78(.72-.83)$ & 620 & 55 & 31 & 3 & 391 & \\
\hline 6 & $24,204.48$ & .65 & $.78(.64-.98)$ & 53 & 595 & 2 & 34 & 370 & 46 \\
\hline \multicolumn{10}{|l|}{ Linear analyses } \\
\hline 1 & $24,254.81$ & NA & 1.0 & 1100 & & & & & \\
\hline 2 & $24,224.64$ & .98 & $.94(.87-.99)$ & 1093 & 7 & & & & \\
\hline 3 & $24,225.76$ & .64 & $.85(.81-.90)$ & 636 & 7 & 457 & & & \\
\hline 4 & $24,228.39$ & .79 & $.84(.80-.90)$ & 993 & 71 & 7 & 30 & & \\
\hline 5 & $24,221.44$ & .63 & $.80(.72-.90)$ & 629 & 331 & 6 & 31 & 103 & \\
\hline 6 & $24,237.72$ & .66 & $.78(.71-.86)$ & 5 & 320 & 32 & 126 & 615 & 2 \\
\hline \multicolumn{10}{|l|}{ lca analyses } \\
\hline 1 & $26,708.06$ & NA & 1.0 & 1100 & & & & & \\
\hline 2 & $25,283.89$ & .79 & $.94(.94-.94)$ & 603 & 497 & & & & \\
\hline 3 & $24,698.63$ & .81 & $.91(.91-.91)$ & 354 & 509 & 237 & & & \\
\hline 4 & $24,504.05$ & .79 & $.88(.86-.90)$ & 229 & 119 & 414 & 338 & & \\
\hline 5 & $24,400.27$ & .78 & $.86(.83-.91)$ & 76 & 288 & 355 & 279 & 102 & \\
\hline 6 & $24,367.06$ & .80 & $.85(.76-.91)$ & 79 & 16 & 352 & 286 & 265 & 102 \\
\hline
\end{tabular}

In bold are the values of the chosen model

BIC Bayesian Information Criterion, NA not applicable, lca latent class analyses 
Fig. 1 Three-trajectory model of HR-QoL $(N=1100)$, based on the general health composite (RAND-12)

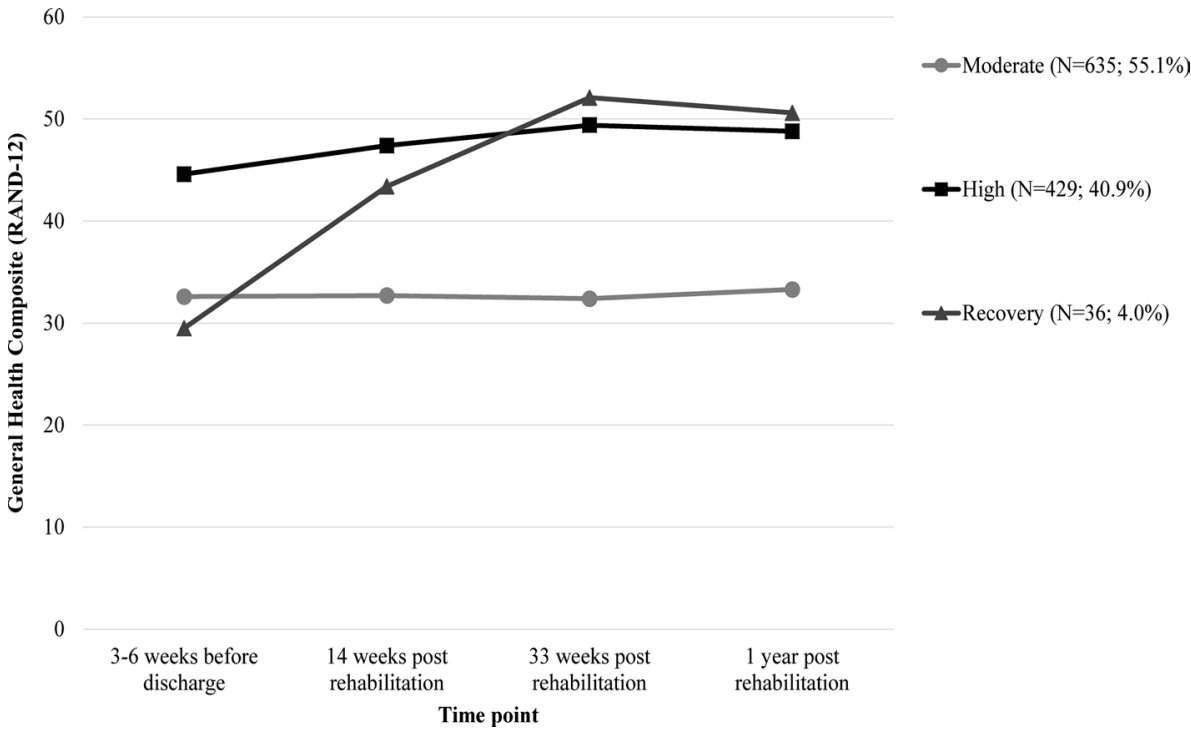

Table 3 Mental, physical and general HR-QoL for the three trajectories at baseline (T0: 3-6 weeks before discharge) and at 14 (T1), 33 (T2) and 52 (T3) weeks after discharge from rehabilitation

\begin{tabular}{lllll}
\hline & T0 & T1 & T2 & T3 \\
& Mean \pm SD & Mean \pm SD & Mean \pm SD & Mean \pm SD \\
\hline Mental health composite & & & & \\
Moderate $(N=635)$ & $36.2 \pm 7.8$ & $36.3 \pm 7.7$ & $35.9 \pm 7.3$ & $37.1 \pm 8.4$ \\
High $(N=429)$ & $46.9 \pm 7.8$ & $49.6 \pm 7.5$ & $51.2 \pm 6.3$ & $49.8 \pm 7.9$ \\
Recovery $(N=36)$ & $35.1 \pm 7.5$ & $46.7 \pm 9.0$ & $55.2 \pm 6.2$ & $53.9 \pm 7.5$ \\
Physical health composite & & & & \\
Moderate $(N=635)$ & $32.0 \pm 8.6$ & $32.0 \pm 8.6$ & $31.9 \pm 8.4$ & $32.2 \pm 8.9$ \\
High $(N=429)$ & $43.1 \pm 8.5$ & $45.4 \pm 8.0$ & $47.3 \pm 7.2$ & $47.5 \pm 7.5$ \\
Recovery $(N=36)$ & $28.2 \pm 10.6$ & $41.1 \pm 11.9$ & $48.3 \pm 8.2$ & $46.9 \pm 9.3$ \\
General health composite & & & & \\
Moderate $(N=635)$ & $32.6 \pm 7.2$ & $32.7 \pm 7.0$ & $32.4 \pm 6.4$ & $33.3 \pm 7.6$ \\
High $(N=429)$ & $44.6 \pm 7.2$ & $47.4 \pm 6.9$ & $49.4 \pm 5.7$ & $48.8 \pm 7.0$ \\
Recovery $(N=36)$ & $29.5 \pm 7.1$ & $43.4 \pm 10.5$ & $52.1 \pm 6.0$ & $50.6 \pm 7.9$ \\
\hline
\end{tabular}

$S D$ standard deviation, $N$ Number of participants

Range: Mental health composite (13-66), Physical health composite (0-63), General health composite $(6-65)$ associations among the personal-, disease- and lifestylerelated factors before discharge from rehabilitation and the HR-QoL trajectories (Table 5).

Compared with participants in the moderate HR-QoL trajectory $(N=635)$, participants with a higher BMI (OR 0.77, 95\% CI 0.64-0.94), participants who perceive fatigue (OR $0.47,95 \%$ CI $0.39-0.58)$ and/or participants who perceive pain (OR 0.22 , 95\% CI 0.15-0.33) are less likely to belong to the latent class with a high HR-QoL trajectory $(N=429)$, while participants who accept their physical disability and/or chronic disease (OR 3.25, 95\% CI 2.25-4.68) are more likely to belong to the latent class with a high HR-QoL trajectory. Also compared to the moderate HR-QoL trajectory, based on the limits of the $95 \%$ CI which both lie above or below one (but not significant), participants who are older (OR 1.27, 95\% CI 1.04-1.55), participants who drink alcohol (OR 1.44, 95\% CI 1.01-2.05) and/or participants who are more physically active (OR $1.21,95 \%$ CI 1.01-1.44) are more likely to belong to the latent class with a high HR-QoL trajectory, while participants who smoke (OR 0.58, 95\% CI $0.35-0.94)$ are less likely to belong to this latent class.

There were no significant determinants before discharge to distinguish between the moderate HR-QoL $(N=635)$ and the recovery HR-QoL $(N=36)$ trajectories. But, based on the limits of the $95 \%$ CI which both lie above one (but not significant), participants who drink alcohol (OR 3.05, 95\% CI 1.09-8.53) are more likely to belong to the latent class 
Table 4 Person-, diseaseand lifestyle-related factors at baseline for the three trajectories of HR-QoL

\begin{tabular}{|c|c|c|c|}
\hline & $\begin{array}{l}\text { Moderate }(N=635) \\
\text { Mean } \pm \text { SD } \\
\text { or } \%(N)\end{array}$ & $\begin{array}{l}\text { High }(N=429) \\
\text { Mean } \pm \text { SD } \\
\text { or } \%(N)\end{array}$ & $\begin{array}{l}\text { Recovery }(N=36) \\
\text { Mean } \pm \mathrm{SD} \\
\text { or } \%(N)\end{array}$ \\
\hline \multicolumn{4}{|l|}{ Personal-related factors } \\
\hline Gender ( $\%$ female) & $57.2(363)$ & 43.6 (187) & $61.1(22)$ \\
\hline Age in years & $50.3 \pm 13.3$ & $52.8 \pm 13.5$ & $42.8 \pm 14.5$ \\
\hline Body mass index $\left(\mathrm{kg} / \mathrm{m}^{2}\right)$ & $27.9 \pm 5.6$ & $26.2 \pm 5.0$ & $27.4 \pm 6.5$ \\
\hline Education level (\% high $)^{\mathrm{a}}$ & $21.3(135)$ & $28.9(124)$ & $30.6(11)$ \\
\hline \multicolumn{4}{|l|}{ Disease-related factors } \\
\hline \multicolumn{4}{|l|}{ Disease group } \\
\hline Musculoskeletal disease & $20.0(127)$ & $13.5(58)$ & $38.9(14)$ \\
\hline Amputation & $2.7(17)$ & $7.5(32)$ & $2.8(1)$ \\
\hline Brain disease & $23.3(148)$ & $30.5(131)$ & $19.4(7)$ \\
\hline Neurologic disease & $17.0(108)$ & $13.5(58)$ & $13.9(5)$ \\
\hline Spinal cord injury & $2.4(15)$ & $3.7(16)$ & $0(0)$ \\
\hline Organ disease & $9.6(61)$ & $15.9(68)$ & $8.3(3)$ \\
\hline Chronic pain & $19.5(124)$ & $10.0(43)$ & $13.9(5)$ \\
\hline Other disease & $3.8(24)$ & 4.4 (19) & $2.8(1)$ \\
\hline Acceptance (\% yes) & $42.0(267)$ & 74.4 (319) & $30.6(11)$ \\
\hline Comorbidities ( $\%$ yes) & $47.1(299)$ & $33.3(143)$ & $33.3(12)$ \\
\hline Fatigue (FSS score) & $4.8 \pm 1.3$ & $3.6 \pm 1.4$ & $4.3 \pm 1.3$ \\
\hline Pain (\% yes) & $60.5(384)$ & $23.3(100)$ & $66.7(24)$ \\
\hline \multicolumn{4}{|l|}{ Lifestyle-related factors } \\
\hline Smoking (\% yes) & $19.4(123)$ & $12.1(52)$ & $13.9(5)$ \\
\hline Alcohol use (\% yes) & $34.6(220)$ & $47.1(202)$ & $22.2(8)$ \\
\hline Total minutes of PA/week & $1031.0 \pm 884.9$ & $1137.6 \pm 956.8$ & $1294.5 \pm 1021.2$ \\
\hline Sports participation ( $\%$ yes) & $52.3(332)$ & $58.5(251)$ & $47.2(17)$ \\
\hline
\end{tabular}

${ }^{\text {a } C o m p l e t e d ~ a p p l i e d ~ U n i v e r s i t y ~ o r ~ h i g h e r ~}$

$S D$ standard deviation, $N$ number of participants, $P A$ physical activity, FSS Fatigue Severity Scale with a moderate HR-QoL trajectory, compared to the recovery HR-QoL trajectory.

A comparison of the recovery HR-QoL trajectory $(N=36)$ and the high HR-QoL trajectory $(N=429)$ showed that participants who are older (OR 1.97, 95\% CI 1.18-3.29), participants who accept their physical disability and/or chronic disease (OR 5.09, 95\% CI 2.04-12.69) and/or participants who drink alcohol (OR 4.60, 95\% CI 1.53-13.83) are more likely to belong to the latent class with a high HR-QoL trajectory $(N=429)$.

Remarkably, gender, education level, type of disease, having comorbidities, level of physical activity and sports participation before discharge were not significant determinants to distinguish between trajectories of HR-QoL.

In addition, we checked whether the found significant determinants in the multiple binomial multivariable logistic regression analyses were still found after controlling for general HR-QoL scores at baseline (Table 5). HR-QoL scores at baseline were found to be significant determinants in the comparisons between the moderate and high HR-QoL trajectories (OR 5.86, 95\% CI 4.14-8.30) and between the recovery and high HR-QoL trajectories (OR 45.24, 95\% CI 10.26-199.47). When controlling for HR-QoL score at baseline, only perceived fatigue (OR $0.69,95 \%$ CI $0.55-0.87$ ) and perceived pain (OR 0.56, 95\% CI 0.35-0.88) remain significant determinants when comparing the moderate and high HR-QoL trajectories (Table 5).

\section{Discussion}

This study identified three distinct trajectories of HR-QoL up to 1 year after rehabilitation in a large heterogeneous cohort of people with a physical disability and/or chronic disease: moderate, high and recovery. The two large and stable trajectories of HR-QoL (moderate and high) among our sample are similar to the large HR-QoL trajectories identified in specific disease populations (e.g. stroke patients [14] and breast cancer survivors [13]), which might indicate that HR-QoL trajectories are not necessarily disease specific. However, we did not identify a decline in HR-QoL trajectory in our sample. Although a considerable group of our sample 


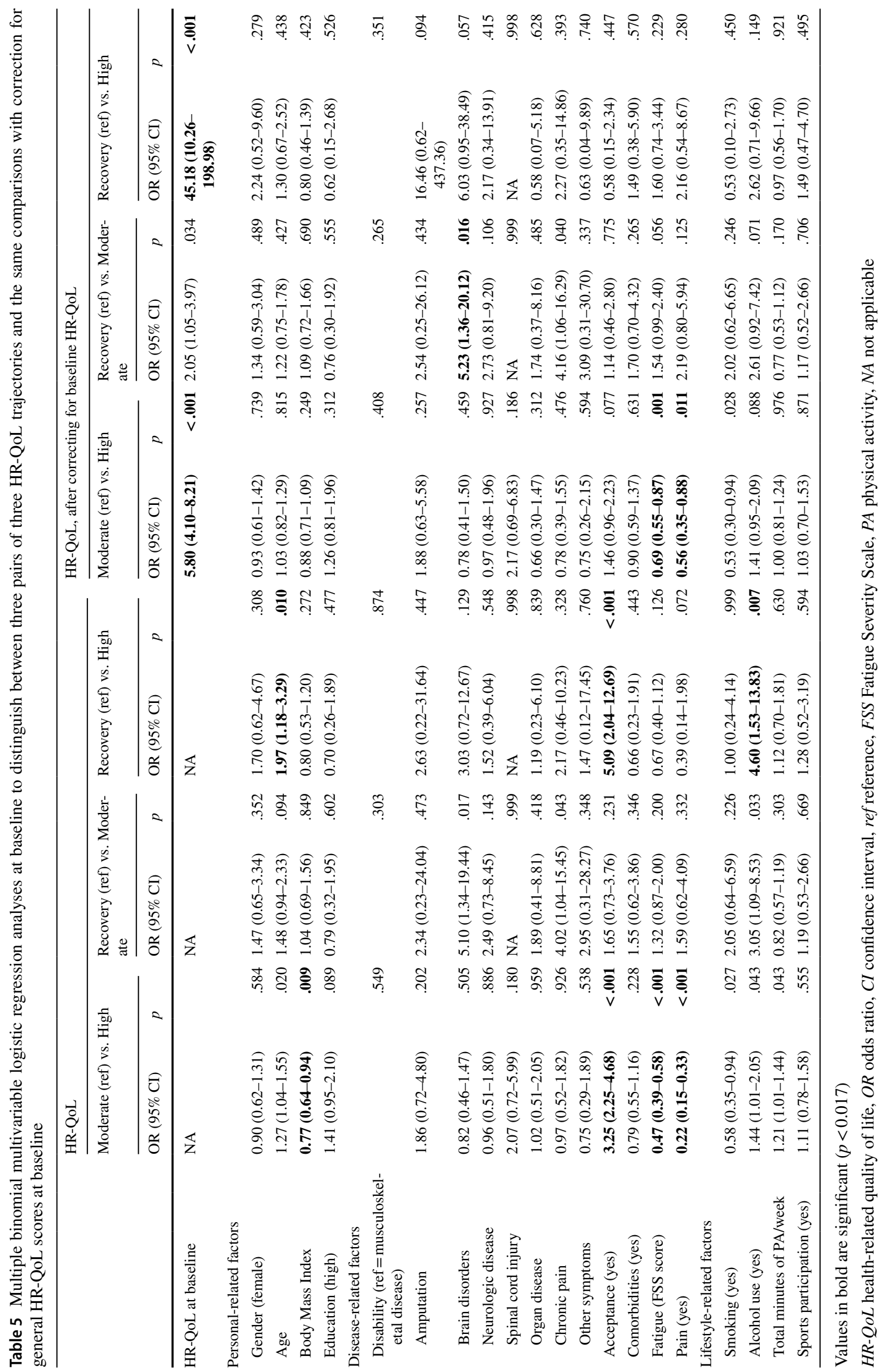


(40.9\%) obtained stable high HR-QoL after participating in the physical activity promotion programme [23, 24], most of the sample (55.1\%) did not.

This study determined which person-, disease- and lifestyle-related factors at discharge from rehabilitation are associated with trajectories of HR-QoL after rehabilitation. The following modifiable disease-related factors were determinants of trajectory membership: acceptance of the disability, perceived fatigue and pain before discharge from rehabilitation. These factors could be explored further for possibilities to modify the vulnerable trajectories into more favourable trajectories of HR-QoL. Acceptance of the disability before discharge from rehabilitation distinguished people in the high HR-QoL trajectory from people in both the moderate and the recovery HR-QoL trajectories. Van Mierlo et al. also found that the acceptance of the disability is a determinant for stable high HR-QoL compared with low HR-QoL in stroke patients [14]. This finding indicates the importance of paying attention to the acceptance of the disability during rehabilitation (e.g. focus on self-management and social/family support [42]), so that people are able to obtain and/or maintain high HR-QoL during and after rehabilitation.

In addition, less perceived fatigue and pain at discharge from rehabilitation strongly distinguishes people in the high HR-QoL trajectory from those in the moderate HR-QoL trajectory, even after controlling for baseline general HR-QoL scores. Fatigue is a distressing secondary health condition that is commonly reported in rehabilitation [43, 44]. Psychological/behavioural treatment (e.g. coping or activity pacing) has been found to be beneficial for reducing fatigue and/ or pain by stimulating a more regular pattern of activities and rest [45], and could play a role in optimising HR-QoL during and after rehabilitation. Activity pacing is a multifaceted coping strategy [46, 47], wherein people who perceive fatigue divide their energy and daily physical activities during the day. Activity pacing can be beneficial for: (1) people at risk of under activity and who are less aware of their energy distribution during the day [48] and (2) people at risk of over activity characterised by an uneven activity pattern consisting of high activity peaks followed by long periods of inactivity [49]. Health care professionals (e.g. sports counsellors or physiotherapists) may improve personcentred advice by motivational interviewing with a focus on activity pacing to reduce perceived fatigue and pain for sustained levels of high HR-QoL after rehabilitation.

Furthermore, we found that 'not consuming alcohol' distinguishes people in the recovery HR-QoL trajectory from people in the high HR-QoL trajectory before discharge. Also, we found confidence that people who do not smoke and/or drink alcohol were more likely to belong to the high HR-QoL trajectory compared to the moderate HR-QoL trajectory, but this finding was not statistically significant. This might be an indication of consequences of unhealthy lifestyle habits, like smoking and alcohol use, not sufficiently addressed during the rehabilitation treatment. More guidance, information and awareness related to general healthy lifestyle behaviours could potentially optimise rehabilitation programmes.

Finally, we did not find physical activity to be statistically significantly associated with HR-QoL trajectories. However, the direction of the association indicates that people who were more physically active before discharge from rehabilitation were more likely to follow the high HR-QoL trajectory compared to people in the moderate HR-QoL trajectory. This might imply that more physical activity is associated with higher HR-QoL, which supports previous literature [7, 9, 50, 51].

Lastly, no significant determinants were found to distinguish between the moderate versus recovery HR-QoL trajectories, probably because these trajectories had comparable HR-QoL scores at baseline. When we control for HR-QoL scores at baseline in the multiple binomial multivariable logistic regression analyses, we see that most significant determinants become non-significant. This implies that especially HR-QoL scores at baseline (the intercepts) of the moderate, high and recovery HR-QoL trajectories can be determined, while most personal-, disease- and lifestylerelated determinants are not able to differentiate between the course (slopes) of the HR-QoL trajectories up to 1 year after discharge from rehabilitation. Only perceived fatigue and pain are still significant determinants to distinguish between the moderate and high HR-QoL trajectories.

Some strengths and limitations of this study need to be addressed. HR-QoL scores (mean \pm standard deviation) found in our cohort before discharge from rehabilitation (physical health: $36.2 \pm 10.3$; mental health: $40.3 \pm 9.4$ ) are comparable to a cohort of primary care patients with chronic diseases (physical health: $36.1 \pm 10.8$; mental health: $40.0 \pm 10.8$ ) [26]. However, HR-QoL scores in our sample are lower compared to people with type 2 diabetes (physical health: $43.5 \pm 10.8$; mental health: $44.8 \pm 10.2$ ) and people after total joint arthroplasty (physical health: $32.1 \pm 8.1$; mental health: 50.0 \pm 9.2 ) [29].

In addition, we used LCGM models to unravel heterogeneity in HR-QoL after rehabilitation and to understand the underlying mechanisms for different subgroups in the population, which has some important advantages. First, this methodological technique categorises people based on their development pattern, a data-driven approach, instead of on a priori classification in theory-driven predefined groups $[35,52]$. Furthermore, this LCGM approach categorises people in homogenous subgroups that represent different profiles of HR-QoL and subsequent health outcomes. This data-driven approach fits with the research design, an observational cohort study, but differs from the traditional way of 
summarising patient data into 'the average patient' [41]. An important point of discussion is the decision on the optimal number of classes, with respect to both the model fit criteria and clinical interpretation. Also, the sample size and the number of measurement occasions have been shown to influence the number and characteristics of the identified classes in the final model [53-56]. Choices made during the modelling process (e.g. model with the lowest BIC) may influence the interpretation of the models and subsequent implications. For example, the five-class quadratic model had a decline HR-QoL trajectory, but also a very small distinct strong recovery HR-QoL trajectory.

In addition, we used the two-step approach to evaluate the characteristics of the latent classes. In step one, we obtained the classes and assigned individuals to their most likely class. In step two, we assessed factors associated with class membership. These steps can also be combined into a one-step approach, where the extra variables are already included in the model during the (conditional) class formation process. Neither approach is right or wrong. The twostep approach for example ignores class assignment error, but does estimate the classes without covariates clouding the class formation $[57,58]$. The one-step approach does incorporate the class assignment uncertainty, but covariates can influence the class formation process $[57,58]$. Our posterior probabilities were relatively high and indicative of low membership error and the one-step approach does not always improve model fit.

Also, we used the RAND-12 questionnaire, which is not preferred over the extended, original RAND-36 questionnaire, nor over more disease-specific HR-QoL questionnaires. However, disease-specific questionnaires were not feasible in our heterogeneous cohort and the shorter RAND12 version provided a solution to the problem to restrict the length of the questionnaire in the ReSpAct study in order to reduce the load for participants [24], which advances the commitment to participate in this longitudinal study.

Furthermore, we found differences between the sample included versus the sample excluded in the current study. Of interest are the acceptance of the disease, fatigue and smoking behaviour. These variables differed statistically significantly between the included and excluded sample as well as between the trajectories. Unfortunately, we were unable to determine the missing at random mechanism, because baseline variables of almost half of the excluded participants were missing.

\section{Implications for practice and research}

More than one third of our sample obtained a relatively stable high HR-QoL, but more than half obtained moderate HRQoL after participating in a person-centred physical activity promotion programme; the RSE programme. We found several modifiable disease-related factors to be important in determining HR-QoL, which emphasises the importance for optimising person-centred advice in focusing on fatigue and pain management and on better acceptance of the disability during rehabilitation. Also, the identified HR-QoL trajectories are not disease specific, which might imply a disease-overarching mechanism.

Furthermore, to make the LCGM modelling more transparent, the data, syntax and results are available in electronic supplementary material. Especially in latent trajectory studies, open communication is important due to the data-driven aspect of the analyses and the difficult choices made to find the optimal model fit. We would like to encourage other researchers in the field of latent trajectory studies, to provide open communication of their analyses and results, and to use the GRoLTS checklist [36] in reporting the analysis of the latent trajectory study. This will benefit comparison of the results in different study populations.

\section{Conclusion}

This study identified three trajectories of HR-QoL after rehabilitation among a large heterogeneous cohort of people with a physical disability and/or chronic disease, of which there were two large stable trajectories (high and moderate), and one small intermediate trajectory (recovery). Our identified HR-QoL trajectories are comparable to HR-QoL trajectories identified in specific disease populations, which might indicate that HR-QoL trajectories are not disease specific. More than half of our sample obtained a relatively stable but moderate HR-QoL after rehabilitation, while $40.9 \%$ obtained a stable high HR-QoL. Membership of these HRQoL trajectories were associated with a limited extend of personal-related factors (age and BMI), disease-related factors (perceived fatigue, perceived pain and acceptance of the disability) and one lifestyle-related factor (alcohol use) before discharge. The moderate HR-QoL trajectory may benefit from person-centred advice during rehabilitation on management of fatigue and pain (e.g. activity pacing), and the acceptance of the disability.

Acknowledgements The authors would like to thank all participants for their contribution to the ReSpAct study. Furthermore, we would like to thank the following organisations for their support in the ReSpAct study: Adelante Zorggroep (Hoensbroek, the Netherlands), Merem behandelcentra, De Trappenberg (Almere, the Netherlands), Vogellanden (Zwolle, the Netherlands), Maasstad Ziekenhuis (Rotterdam, the Netherlands), Noordwest Ziekenhuisgroep (Alkmaar, the Netherlands), Militair Revalidatiecentrum Aardenburg (Doorn, the Netherlands), Rehabilitation Center Leijpark (Tilburg, the Netherlands), Rehabilitation Center Reade (Amsterdam, the Netherlands), Revalidatie Friesland (Heerenveen, the Netherlands), Revant (Breda, the Netherlands), Rijnlands Rehabilitation Center (Leiden, the Netherlands), Klimmendaal (Arnhem, the Netherlands), Treant Zorggroep (Hoogeveen and Emmen, 
the Netherlands), Sint Maartenskliniek (Nijmegen, the Netherlands), Sophia Rehabilitation Center (Den Haag, the Netherlands), Tolbrug Rehabilitation ('s Hertogenbosch, the Netherlands), Klimmendaal, Sport Variant (Apeldoorn, the Netherlands). The authors would like to thank Leonie A. Krops and Pim Brandenbarg for their critical reading and comments on a draft of the manuscript.

Funding This study was funded by the Dutch Ministry of Health, Welfare and Sports (Grant No. 319758), Stichting Beatrixoord NoordNederland (grant date 19-2-2018) and a personal grant received from the University Medical Center Groningen, and supported by the Knowledge Center of Sport Netherlands and Stichting Special Heroes Nederland (before January 2016: Stichting Onbeperkt Sportief).

\section{Compliance with ethical standards}

Conflict of interest The authors declare that they have no conflicts of interest or financial disclosures.

Ethical approval All procedures performed in studies involving human participants were in accordance with the ethical standards of the institutional research committee and with the 1964 Helsinki declaration and its later amendments or comparable ethical standards. The study was approved by the ethics committee of the Center for Human Movement Sciences of the University Medical Center Groningen (reference: ECB/2013.02.28_1).

Informed consent All individual participants included in the study provided written informed consent.

Open Access This article is licensed under a Creative Commons Attribution 4.0 International License, which permits use, sharing, adaptation, distribution and reproduction in any medium or format, as long as you give appropriate credit to the original author(s) and the source, provide a link to the Creative Commons licence, and indicate if changes were made. The images or other third party material in this article are included in the article's Creative Commons licence, unless indicated otherwise in a credit line to the material. If material is not included in the article's Creative Commons licence and your intended use is not permitted by statutory regulation or exceeds the permitted use, you will need to obtain permission directly from the copyright holder. To view a copy of this licence, visit http://creativecommons.org/licenses/by/4.0/.

\section{References}

1. Hays, R. D., Hahn, H., \& Marshall, G. (2002). Use of the SF-36 and other health-related quality of life measures to assess persons with disabilities. Archives of Physical Medicine and Rehabilitation, 83(12 Suppl 2), S4-9.

2. Gellert, G. A. (1993). The importance of quality of life research for health care reform in the USA and the future of public health. Quality of Life Research, 2(5), 357-361.

3. Schrier, E., Schrier, I., Geertzen, J. H., \& Dijkstra, P. U. (2016). Quality of life in rehabilitation outpatients: Normal values and a comparison with the general dutch population and psychiatric patients. Quality of Life Research, 25(1), 135-142.

4. Rimmer, J. H. (2012). Getting beyond the plateau: Bridging the gap between rehabilitation and community-based exercise. $P M$ \& $R, 4(11), 857-861$

5. Estrella-Castillo, D. F. P. D., \& Gómez-de-Regil, L. P. D. (2016). Quality of life in Mexican patients with primary neurological or musculoskeletal disabilities. Disability and Health Journal, 9(1), 127-133.

6. Kaske, S., Lefering, R., Trentzsch, H., Driessen, A., Bouillon, B., Maegele, M., et al. (2014). Quality of life two years after severe trauma: A single-centre evaluation. Injury, 45(Suppl 3), S100-S105.

7. Krops, L. A., Jaarsma, E. A., Dijkstra, P. U., Geertzen, J. H., \& Dekker, R. (2017). Health related quality of life in a dutch rehabilitation population: Reference values and the effect of physical activity. PLOS ONE, 12(1), e0169169.

8. Rimmer, J. H., Chen, M. D., \& Hsieh, K. (2011). A conceptual model for identifying, preventing, and managing secondary conditions in people with disabilities. Physical Therapy, 91(12), 1728-1739.

9. Feeny, D., Garner, R., Bernier, J., Thompson, A., McFarland, B. H., Huguet, N., et al. (2014). Physical activity matters: Associations among body mass index, physical activity, and health-related quality of life trajectories over 10 years. Journal of Physical Activity and Health, 11(7), 1265-1275.

10. Sawatzky, R., Liu-Ambrose, T., Miller, W. C., \& Marra, C. A. (2007). Physical activity as a mediator of the impact of chronic conditions on quality of life in older adults. Health and Quality Life Outcomes, 5, 68.

11. Haskell, W. L., Lee, I. M., Pate, R. R., Powell, K. E., Blair, S. N., Franklin, B. A., et al. (2007). Physical activity and public health: Updated recommendation for adults from the american college of sports medicine and the american heart association. Circulation, 116(9), 1081-1093.

12. Carroll, D. D., Courtney-Long, E. A., Stevens, A. C., Sloan, M. L., Lullo, C., Visser, S. N., et al. (2014). Vital signs: Disability and physical activity-United States, 2009-2012. MMWR Morbidity and Mortality Weekly Report, 63(18), 407-413.

13. Goyal, N. G., Levine, B. J., Van Zee, K. J., Naftalis, E., \& Avis, N. E. (2018). Trajectories of quality of life following breast cancer diagnosis. Breast Cancer Research Treatment, 169, 163-173.

14. van Mierlo, M., van Heugten, C., Post, M. W. M., Hoekstra, T., \& Visser-Meily, A. (2017). Trajectories of health-related quality of life after stroke: Results from a one-year prospective cohort study. Disability and Rehabilitation, 13, 1-10.

15. Bonanno, G. A., \& Mancini, A. D. (2008). The human capacity to thrive in the face of potential trauma. Pediatrics, 121(2), 369-375.

16. Lee, D. T., Choi, K. C., Chair, S. Y., Yu, D. S., \& Lau, S. T. (2014). Psychological distress mediates the effects of socio-demographic and clinical characteristics on the physical health component of health-related quality of life in patients with coronary heart disease. European Journal of Preventive Cardiology, 21(1), 107-116.

17. Passier, P. E., Visser-Meily, J. M., van Zandvoort, M. J., Rinkel, G. J., Lindeman, E., \& Post, M. W. (2012). Predictors of long-term health-related quality of life in patients with aneurysmal subarachnoid hemorrhage. NeuroRehabilitation, 30(2), 137-145.

18. Teoh, V., Sims, J., \& Milgrom, J. (2009). Psychosocial predictors of quality of life in a sample of community-dwelling stroke survivors: A longitudinal study. Topics in Stroke Rehabilitation, 16(2), 157-166.

19. Visser-Meily, J. M., Rhebergen, M. L., Rinkel, G. J., van Zandvoort, M. J., \& Post, M. W. (2009). Long-term health-related quality of life after aneurysmal subarachnoid hemorrhage: Relationship with psychological symptoms and personality characteristics. Stroke, 40(4), 1526-1529.

20. Beisland, E., Beisland, C., Hjelle, K. M., Bakke, A., Aarstad, A. K., \& Aarstad, H. J. (2015). Health-related quality of life, personality and choice of coping are related in renal cell carcinoma patients. Scandinavian Journal of Urology, 49(4), 282-289.

21. Grauwmeijer, E., Heijenbrok-Kal, M. H., \& Ribbers, G. M. (2014). Health-related quality of life 3 years after moderate to 
severe traumatic brain injury: A prospective cohort study. Archives of Physical Medicine and Rehabilitation, 95(7), 1268-1276.

22. White, G. W., Gonda, C., Peterson, J. J., Drum, C. E., \& RRTC Expert Panel on Health Promotion Interventions. (2011). Secondary analysis of a scoping review of health promotion interventions for persons with disabilities: Do health promotion interventions for people with mobility impairments address secondary condition reduction and increased community participation? Disability and Health Journal, 4(2), 129-139.

23. Hoekstra, F., Alingh, R. A., van der Schans, C. P., Hettinga, F. J., Duijf, M., Dekker, R., et al. (2014). Design of a process evaluation of the implementation of a physical activity and sports stimulation programme in dutch rehabilitation setting: ReSpAct. Implementation Science, 9, 127.

24. Alingh, R. A., Hoekstra, F., van der Schans, C. P., Hettinga, F. J., Dekker, R., \& van der Woude, L. H. (2015). Protocol of a longitudinal cohort study on physical activity behaviour in physically disabled patients participating in a rehabilitation counselling programme: ReSpAct. British Medical Journal Open, 5(1), e007591.

25. Miller, W. R., \& Rose, G. S. (2009). Toward a theory of motivational interviewing. American Psychologist, 64(6), 527-537.

26. Feeny, D., Farris, K., Cote, I., Johnson, J. A., Tsuyuki, R. T., \& Eng, K. (2005). A cohort study found the RAND-12 and health utilities index mark 3 demonstrated construct validity in high-risk primary care patients. Journal of Clinical Epidemiology, 58(2), $138-141$.

27. Hays, R. D., \& Morales, L. S. (2001). The RAND-36 measure of health-related quality of life. Annals of Medicine, 33(5), 350-357.

28. Ware, J., Jr., Kosinski, M., \& Keller, S. D. (1996). A 12-item short-form health survey: Construction of scales and preliminary tests of reliability and validity. Medical Care, 34(3), 220-233.

29. Maddigan, S. L., Feeny, D. H., Johnson, J. A., \& DOVE Investigators. (2004). Construct validity of the RAND-12 and health utilities index mark 2 and 3 in type 2 diabetes. Quality of Life Research, 13(2), 435-448.

30. Krupp, L. B., LaRocca, N. G., Muir-Nash, J., \& Steinberg, A. D. (1989). The fatigue severity scale: Application to patients with multiple sclerosis and systemic lupus erythematosus. Archives of Neurology, 46(10), 1121-1123.

31. Whitehead, L. (2009). The measurement of fatigue in chronic illness: A systematic review of unidimensional and multidimensional fatigue measures. Journal of Pain and Symptom Management, 37(1), 107-128.

32. Elbers, R. G., Rietberg, M. B., van Wegen, E. E., Verhoef, J., Kramer, S. F., Terwee, C. B., et al. (2012). Self-report fatigue questionnaires in multiple sclerosis, parkinson's disease and stroke: A systematic review of measurement properties. Quality of Life Reearch, 21(6), 925-944.

33. Seves, B. L., Hoekstra, F., Schoenmakers, J. W., Brandenbarg, P., Hoekstra, T., Hettinga, F. J., et al. (2020). Test-retest reliability and concurrent validity of the adapted short QUestionnaire to ASsess health-enhancing physical activity (adaptedSQUASH) in adults with disabilities. medRxiv. https://doi. org/10.1101/2020.09.09.20190371.

34. Wendel-Vos, G. C., Schuit, A. J., Saris, W. H., \& Kromhout, D. (2003). Reproducibility and relative validity of the short questionnaire to assess health-enhancing physical activity. Journal of Clinical Epidemiology, 56(12), 1163-1169.

35. Jung, T., \& Wickrama, K. A. S. (2008). An introduction to latent class growth analysis and growth mixture modeling. Social and Personality Psychology Compass, 2(1), 302-317.

36. van de Schoot, R., Sijbrandij, M., Winter, S. D., Depaoli, S., \& Vermunt, J. K. (2017). The GRoLTS-checklist: Guidelines for reporting on latent trajectory studies. Structural Equation Modeling: A Multidisciplinary Journal, 24(3), 451-467.
37. Raftery, A. E. (1995). Bayesian model selection in social research. Sociological Methodology, 25, 111.

38. Ramaswamy, V., Desarbo, W. S., Reibstein, D. J., \& Robinson, W. T. (1993). An empirical pooling approach for estimating marketing mix elasticities with PIMS data. Marketing Science, 12(1), 103-124.

39. Carragher, N., Adamson, G., Bunting, B., \& McCann, S. (2009). Subtypes of depression in a nationally representative sample. Journal of Affective Disorders, 113(1-2), 88-99.

40. Brown, B. W., \& Russell, K. (1997). Methods correcting for multiple testing: Operating characteristics. Statistics in Medicine, 16(22), 2511-2528.

41. Hoekstra, T. (2013). Applied latent class models for epidemiology (dissertation). Amsterdam: VU University.

42. Li, L., \& Moore, D. (1998). Acceptance of disability and its correlates. Journal of Social Psychology, 138(1), 13-25.

43. Clark, L. V., \& White, P. D. (2009). The role of deconditioning and therapeutic exercise in chronic fatigue syndrome (CFS). Journal of Mental Health, 14(3), 237-252.

44. Nijs, J., Paul, L., \& Wallman, K. (2008). Chronic fatigue syndrome: An approach combining self-management with graded exercise to avoid exacerbations. Journal of Rehabilitation Medicine, 40(4), 241-247.

45. Acciarresi, M., Bogousslavsky, J., \& Paciaroni, M. (2014). Poststroke fatigue: Epidemiology, clinical characteristics and treatment. European Neurology, 72(5-6), 255-261.

46. Antcliff, D., Keeley, P., Campbell, M., Woby, S., Keenan, A. M., \& McGowan, L. (2018). Activity pacing: Moving beyond taking breaks and slowing down. Quality of Life Research, 27, 1933-1935.

47. Abonie, U. S., Sandercock, G. R. H., Heesterbeek, M., \& Hettinga, F. J. (2018). Effects of activity pacing in patients with chronic conditions associated with fatigue complaints: A meta-analysis. Disability and Rehabilitation, 18, 1-10.

48. Gill, J. R., \& Brown, C. A. (2009). A structured review of the evidence for pacing as a chronic pain intervention. European Journal of Pain, 13(2), 214-216.

49. Antcliff, D., Keeley, P., Campbell, M., Oldham, J., \& Woby, S. (2013). The development of an activity pacing questionnaire for chronic pain and/or fatigue: A delphi technique. Physiotherapy, 99(3), 241-246.

50. Marck, C. H., Hadgkiss, E. J., Weiland, T. J., van der Meer, D. M., Pereira, N. G., \& Jelinek, G. A. (2014). Physical activity and associated levels of disability and quality of life in people with multiple sclerosis: A large international survey. BMC Neurology, 14, 143.

51. Farris, M. S., Kopciuk, K. A., Courneya, K. S., McGregor, S. E., Wang, Q., \& Friedenreich, C. M. (2017). Identification and prediction of health-related quality of life trajectories after a prostate cancer diagnosis. International Journal of Cancer, 140(7), $1517-1527$.

52. Muthen, B. (2006). The potential of growth mixture modelling. Infant and Child Development, 15(6), 623-625.

53. Connell, A. M., \& Frye, A. A. (2006). Response to commentaries on target paper, 'Growth mixture modeling in developmental psychology'. Infant and Child Development, 15(6), 639-642.

54. Stanger, C. (2006). Latent growth mixture models: An important new tool for developmental researchers. Infant and Child Development, 15(6), 635-637.

55. Bauer, D. J., \& Curran, P. J. (2003). Distributional assumptions of growth mixture models: Implications for overextraction of latent trajectory classes. Psychological Methods, 8(3), 338-363.

56. Nagin, D. S., \& Tremblay, R. E. (2001). Analyzing developmental trajectories of distinct but related behaviors: A group-based method. Psychological Methods, 6(1), 18-34. 
57. Huang, D., Brecht, M. L., Hara, M., \& Hser, Y. I. (2010). Influences of a covariate on growth mixture modeling. J Drug Issues Winter, 40(1), 173-194.

58. Muthén, B. (2003). Statistical and substantive checking in growth mixture modeling: Comment on bauer and curran (2003). Psychological Methods, 8(3), 369-377.
Publisher's Note Springer Nature remains neutral with regard to jurisdictional claims in published maps and institutional affiliations. 